INT-PUB-11-054

\title{
Strangelet dwarfs
}

\author{
Mark G. Alford, Sophia Han (韩君) \\ Physics Department, Washington University, St. Louis, MO 63130, USA \\ Sanjay Reddy \\ Institute for Nuclear Theory, University of Washington, Seattle, Washington 98195-1550, USA
}

(Dated: 7 March 2012)

\begin{abstract}
If the surface tension of quark matter is low enough, quark matter is not self bound. At sufficiently low pressure and temperature, it will take the form of a crystal of positively charged strangelets in a neutralizing background of electrons. In this case there will exist, in addition to the usual family of strange stars, a family of low-mass large-radius objects analogous to white dwarfs, which we call "strangelet dwarfs". Using a generic parametrization of the equation of state of quark matter, we calculate the mass-radius relationship of these objects.
\end{abstract}

PACS numbers: $25.75 . \mathrm{Nq}, 97.20 . \mathrm{Rp}, 26.60 .-\mathrm{c}, 97.60 . \mathrm{Jd}$,

\section{INTRODUCTION}

The matter that is directly observed in nature consists of atoms, whose nuclei are droplets of nuclear matter composed of up and down quarks. Nuclear matter is very stable: the most stable nuclei have lifetimes longer than the age of the universe. However, it has been hypothesized [1-3] that nuclear matter may actually be metastable, and the true ground state of matter consists of a combination of roughly equal numbers of up, down, and strange quarks known as "strange matter". Strange matter is hypothesized to exist as (kilometersized) pieces, known as "strange stars" (reviewed in [4), or as small nuggets, known as "strangelets" 3. It has further been hypothesized that dark matter could be some form of quark matter, trapped in strangelets or strange stars before the era of nucleosynthesis [5-7]. However, even if strange matter is not invoked as a dark matter candidate, there could still be a population of strange matter objects, from strange stars to strangelets, many of which would be relatively non-luminous. In this article we show that the masses and radii of such objects can extend in to the range expected for planets. Recent surveys such as the Microlensing Observations in Astrophysics (MOA) and the Optical Gravitational Lensing Experiment (OGLE) to detect such low mass non-luminious low mass objects by gravitational lensing have yielded interesting results 8 . The hypothetical compact objects we predict could be detected by such methods and such surveys could place stringent bounds or perhaps hint at their possible existence.

It is generally assumed that strange stars are compact objects, with sizes in the 10 kilometer range 4], ending at a sharp surface of thickness $\sim 1$ Fermi, perhaps with a very thin electrostatically suspended nuclear matter crust 9 11]. However, if the surface tension $\sigma$ of the interface between quark matter and the vacuum is less than a critical value $\sigma_{\text {crit }}$ (of order a few $\mathrm{MeV} / \mathrm{fm}^{2}$ in typical models of quark matter) then large strangelets are unstable against fission into smaller ones [12 14], and the energetically preferred state is a crystal of strangelets: a mixed phase consisting of nuggets of positively-charged strange matter in a neutralizing background of electrons.

In this "low surface tension" scenario, strange stars are not self-bound: they require gravitational attraction to bind the strangelets. Stars made of strange matter are then qualitatively similar to those made of nuclear matter: in each case the mass-radius relation has two branches, one compact and the other diffuse. For nuclear matter, the compact branch contains neutron stars, which consist of gravitationally bound nuclear matter, with an outer crust that is a crystal of nuclei in a background of electrons; the diffuse branch contains white dwarfs, which are a gravitationally bound cold plasma of nuclei (ions) and electrons, forming, at sufficiently low temperature, a crystalline structure (see, e.g., [15]). For strange matter with a low surface tension, there are similarly two branches. The compact branch contains strange stars with a crust that consists of strangelets in a background of electrons; this "strangelet crystal crust" was studied in Ref. [14]. In this paper we study the diffuse branch, which has no core of uniform quark matter: these stars consist entirely of strangelets in a background of degenerate electrons, so by analogy with white dwarfs we call them strangelet dwarfs.

The strangelet-crystal phase is a charge-separated phase. Charge separation is favored by the internal energy of the phases involved, because a neutral phase is always at a maximum of the free energy with respect to the electrostatic potential (see [16, 17; for a pedagogical discussion see [18]). The domain structure is determined by competition between surface tension (which favors large domains) and electric field energy (which favors small domains). Debye screening plays a role in determining the domain structure, because it redistributes the electric charge, concentrating it in the outer part of the quark matter domains and the inner part of the surrounding electron gas, and thereby modifying the internal energy and electrostatic energy contributions. Our parameterization (1) of the electrostatic properties of quark matter is generic, but is not appropriate for strangelets in the color-flavor locked (CFL) phase [19, which is a degener- 
ate case requiring separate treatment (see Sec. IV A.

To obtain the $M(R)$ relation of strangelet dwarfs, we solve the Tolman Oppenheimer Volkoff equation [20, 21, using the equation of state of the mixed phase. We obtain the equation of state by assuming that the strangelet lattice can be divided into unit cells ("Wigner-Seitz cells") and calculating the pressure of a cell as a function of its energy density. Our approach is similar to that used in previous studies of the strangelet crystal [13, 14] (except that in this paper we include electron mass effects) and in studies of mixed phases of quark matter and nuclear matter in the interior of neutron stars [22].

The main assumptions that we make are:

1) We assume that the strangelets in the plasma form a regular lattice of Wigner-Seitz cells, which we treat as rotationally invariant (spherical). In reality the cells will be unit cells of some regular lattice. We do not consider lower-dimensional structures (rods or slabs) because in Ref. [14] we found that such structures were never energetically favored.

2) Within each Wigner-Seitz cell we use a Thomas-Fermi approach, solving the Poisson equation to obtain the charge distribution, energy density, and pressure. This is incorrect for very small strangelets, where the energy level structure of the quarks becomes important [23, 24]. 3) We treat the interface between quark matter and the vacuum as a sharp interface which is characterized by a surface tension. We assume there is no charge localized on the surface. (Thus we neglect any surface charge that might arise from the reduction of the density of states of strange quarks at the surface [25 28].)

4) We neglect the curvature energy of a quark matter surface [29, 30], so we do not allow for "Swiss-cheese" mixed phases, in which the outer part of the Wigner-Seitz cell is filled with quark matter, with a cavity in the center, for which the curvature energy is crucial.

5) We work at zero temperature.

In our calculations we use units $\hbar=c=\epsilon_{0}=1$, so $\alpha=e^{2} /(4 \pi) \approx 1 / 137$.

\section{PHENOMENOLOGICAL DESCRIPTION OF QUARK MATTER}

We use the fact that in most phases of quark matter the chemical potential for negative electric charge $\mu_{e}$ is much less than the chemical potential for quark number $\mu$. This allows us to write down a model-independent parameterization of the quark matter equation of state, expanded in powers of $\mu_{e} / \mu$ [13],

$$
p_{\mathrm{QM}}\left(\mu, \mu_{e}\right) \approx p_{0}(\mu)-n_{Q}(\mu) \mu_{e}+\frac{1}{2} \chi_{Q}(\mu) \mu_{e}^{2}+\ldots
$$

Note that the contribution of electrons to the pressure of quark matter is $\mathcal{O}\left(\mu_{e}^{4}\right)$, and is neglected. This is a very good approximation for small strange quark mass, which corresponds to small $n_{Q}$. (For the largest value of $n_{Q}$ that we study, $\mu_{e}$ in neutral quark matter is close to $100 \mathrm{MeV}$, and the assumption is still reasonable.)
As noted in Sec. [1, we assume that the interface between quark matter and vacuum has a surface tension $\sigma$, and we neglect any curvature energy.

The quark density $n$ and the electric charge density $q_{\mathrm{QM}}$ (in units of the positron charge) are

$$
n=\frac{\partial p_{\mathrm{QM}}}{\partial \mu}, \quad q_{\mathrm{QM}}=-\frac{\partial p_{\mathrm{QM}}}{\partial \mu_{e}}=n_{Q}-\chi_{Q} \mu_{e} .
$$

So in uniform neutral quark matter the electron chemical potential is $\mu_{e}^{\text {neutral }}=n_{Q} / \chi_{Q}$. Eq. (1) is a generic parametrization if $\mu_{e}^{\text {neutral }} \ll \mu$, which is typically the case in three-flavor quark matter.

The bag constant enters in $p_{0}(\mu)$, and we will fix it by requiring that the first-order transition between neutral quark matter and the vacuum occur at quark chemical potential $\mu_{\text {crit }}$, i.e. $p\left(\mu_{\text {crit }}, \mu_{e}^{\text {neutral }}\right)=0$. Because we are assuming that the strange matter hypothesis is valid, we require $\mu_{\text {crit }} \lesssim 310 \mathrm{MeV}$, since at $\mu \approx 310 \mathrm{MeV}$ there is a transition from vacuum to neutral nuclear matter. In this article we will typically use $\mu_{\text {crit }}=300 \mathrm{MeV}$. The value of $\mu$ inside our quark matter lumps will always be very close to $\mu_{\text {crit }}$, so we can also expand in powers of $\mu-\mu_{\text {crit }}$, and write

$$
\begin{aligned}
p_{\mathrm{QM}}\left(\mu, \mu_{e}\right) \approx & n\left(\mu-\mu_{\mathrm{crit}}\right)+\frac{1}{2} \chi\left(\mu-\mu_{\mathrm{crit}}\right)^{2} \\
& +\frac{n_{Q}^{2}}{2 \chi_{Q}}-n_{Q} \mu_{e}+\frac{1}{2} \chi_{Q} \mu_{e}^{2} .
\end{aligned}
$$

A quark matter equation of state can then be expressed in terms of 6 numbers: $\mu_{\text {crit }}$, the charge density $n_{Q}$ and charge susceptibility $\chi_{Q}$ evaluated at $\mu=\mu_{\text {crit }}$, the quark number density $n$ and susceptibility $\chi$ evaluated at $\mu=$ $\mu_{\text {crit }}$, and the surface tension $\sigma$.

We will restrict ourselves to values of the surface tension that are below the critical value [13]

$$
\sigma_{\text {crit }}=0.1325 \frac{n_{Q}^{2} \lambda_{D}}{\chi_{Q}}=0.1325 \frac{n_{Q}^{2}}{\sqrt{4 \pi \alpha} \chi_{Q}^{3 / 2}},
$$

where $\lambda_{D}$ is the Debye screening length in quark matter

$$
\lambda_{D}=\frac{1}{\sqrt{4 \pi \alpha \chi_{Q}}} .
$$

If the surface tension is larger than $\sigma_{\text {crit }}$ then the energetically favored structure at low pressure will not be a strangelet crystal, and there will be no strangelet dwarfs. Rough estimates of surface tension from the bag model are in the range 4 to $10 \mathrm{MeV} / \mathrm{fm}^{2}$ [31, 32, and for typical models of quark matter, $\sigma_{\text {crit }}$ is of order 1 to $10 \mathrm{MeV} / \mathrm{fm}^{2}$ [13], so it is reasonable to explore the possibility that strange quark matter could have a surface tension below $\sigma_{\text {crit }}$.

\section{A. Specific equations of state}

When we show numerical results we will need to vary $n_{Q}$ and $\chi_{Q}$ over a range of physically reasonable values. 
To give a rough idea of what values are appropriate, we consider the example of non-interacting three-flavor quark matter, for which $n_{Q}$ and $\chi_{Q}$ become functions of $\mu$ and the strange quark mass $m_{s}$, while $p_{0}$ is in addition a function of the bag constant $B$. Expanding to lowest non-trivial order in $m_{s}$,

$$
\begin{aligned}
p_{0}(\mu) & =\frac{9 \mu^{4}}{12 \pi^{2}}-B, \\
n_{Q}\left(\mu, m_{s}\right) & =\frac{m_{s}^{2} \mu}{2 \pi^{2}}, \\
\chi_{Q}\left(\mu, m_{s}\right) & =\frac{2 \mu^{2}}{\pi^{2}} .
\end{aligned}
$$

We emphasize that these expressions are simply meant to give a rough idea of reasonable physical values for $n_{Q}$ and $\chi_{Q}$. Our treatment does not depend on an expansion in powers of $m_{s}$. To tune the transition between neutral quark matter and the vacuum so it occurs at $\mu=\mu_{\text {crit }}$ (see previous subsection), we set $B$ so that $p_{0}\left(\mu_{\text {crit }}\right)=$ $\frac{1}{2} n_{Q}^{2}\left(\mu_{\text {crit }}\right) / \chi_{Q}\left(\mu_{\text {crit }}\right)$.

In the regions between lumps of strange matter, we will assume that there is a degenerate electron gas, whose pressure, and charge density in units of $e$, are

$$
\begin{aligned}
p_{e^{-}}\left(\mu_{e}\right)= & \frac{1}{24 \pi^{2}}\left(\left(2 k_{F e}^{2}-3 m^{2}\right) k_{F e} \mu_{e}\right. \\
& \left.+3 m^{4} \ln \left(\frac{k_{F e}+\mu_{e}}{m}\right)\right), \\
q_{e^{-}}\left(\mu_{e}\right)= & -\frac{1}{3 \pi^{2}} k_{F e}^{3} .
\end{aligned}
$$

where $\mu_{e}^{2}=k_{F e}^{2}+m_{e}^{2}$. Note that at low pressures this is more accurate than the electron gas equation of state used in Ref. [14, where the electron mass was set to zero.

\section{EQUATION OF STATE OF STRANGELET CRYSTAL}

\section{A. Wigner-Seitz cell}

Following the approach of [14, we analyze a spherical Wigner-Seitz cell of radius $R_{\text {cell }}$, with a sphere of quark matter at the center of radius $R$. We use the ThomasFermi approximation to calculate $\mu_{e}(r)$,

$$
\nabla^{2} \mu_{e}(r)=-4 \pi \alpha q(r)
$$

where $q(r)$ is the electric charge density in units of the positron charge $e$, and $\mu_{e}$ is the electrostatic potential divided by $e$.

The boundary conditions are that there is no electric field in the center of the cell (no $\delta$-function charge there), and no electric field at the edge of the cell (the cell is electrically neutral),

$$
\frac{d \mu_{e}}{d r}(0)=0, \quad \frac{d \mu_{e}}{d r}\left(R_{\text {cell }}\right)=0
$$

We also need a matching condition at the edge of the quark matter. Since we assume that no charge is localized on the surface, we require continuity of $\mu_{e}$ and its first derivative (the electric field) at $r=R$.

The value of $\mu$ inside the strange matter will be slightly different from $\mu_{\text {crit }}$ because the surface tension compresses the droplet. To determine the value of $\mu$, we require the pressure discontinuity across the surface of the strangelet to be balanced by the surface tension:

$$
p_{\mathrm{QM}}\left(\mu, \mu_{e}(R)\right)-p_{e^{-}}\left(\mu_{e}(R)\right)=\frac{2 \sigma}{R} .
$$

Once these equations are solved, we can obtain the equation of state of matter made of such cells. The total energy of a cell is

$$
\begin{aligned}
E & =4 \pi \int_{0}^{R} r^{2} d r\left(\mu n\left(\mu_{e}\right)-\frac{1}{2} \mu_{e} q_{\mathrm{QM}}\left(\mu_{e}\right)-p_{\mathrm{QM}}\left(\mu, \mu_{e}\right)\right) \\
& +4 \pi \int_{R}^{R_{\mathrm{cell}}} r^{2} d r\left(-\frac{1}{2} \mu_{e} q_{e^{-}}\left(\mu_{e}\right)-p_{e^{-}}\left(\mu_{e}\right)\right) \\
& +4 \pi R^{2} \sigma,
\end{aligned}
$$

The $-\frac{1}{2} \mu_{e} q$ terms in 11 come from combining $-\mu_{e} q$ (from the relationship between energy density and pressure) with the electric field energy density $+\frac{1}{2} \mu_{e} q$. The pressure of the cell is simply the pressure of the electrons at the edge of the cell,

$$
p_{\text {cell }}=p_{e^{-}}\left(\mu_{e}\left(R_{\text {cell }}\right)\right) .
$$

The total number of quarks is

$$
N=4 \pi \int_{0}^{R} r^{2} d r n\left(\mu, \mu_{e}\right) .
$$

The volume of the cell is $V=(4 / 3) \pi R_{\text {cell }}{ }^{3}$.

By varying $R$ and $R_{\text {cell }}$ we generate a two-parameter family of strangelets. However, there is really only a single-parameter family of physical configurations, parameterized by the external pressure $p_{\text {cell }}$. On each line of constant $p_{\text {cell }}$ in the $\left(R, R_{\text {cell }}\right)$ parameter space, we must minimize the enthalpy per quark,

$$
h=\frac{E+p_{\text {cell }} V}{N},
$$

to find the favored value of $R$ and $R_{\text {cell }}$. We assume zero temperature so $h$ is also the Gibbs free energy per quark.

We now have a well-defined way to obtain the equation of state of the mixed phase of quark matter, namely the energy density $\varepsilon=E / V$ as a function of the pressure $p_{\text {cell }}$.

\section{B. Numerical solution}

Inside the quark matter, the solution to the Poisson equation (8) that obeys the boundary condition at the 
origin is

$$
\mu_{e}(r)=\frac{n_{Q}}{\chi_{Q}}+\frac{A}{r \lambda_{D}} \sinh \left(\frac{r}{\lambda_{D}}\right)
$$

where $A$ will be determined by matching conditions.

In the degenerate electron gas region outside the strange matter, from (7) and (8) the Poisson equation becomes

$$
\nabla^{2} \mu_{e}(r)=\frac{4 \alpha}{3 \pi}\left(\mu_{e}^{2}-m_{e}^{2}\right)^{3 / 2}
$$

which must be solved numerically. For a given value of $A$ we find from (15) the value and slope of $\mu_{e}(r)$ at $r=R$, and use these as initial values to propagate $\mu_{e}(r)$ out to $r=R_{\text {cell }}$ using 16 . We vary $A$ until we obtain a solution that obeys the boundary condition of no electric field at the edge of the cell.

\section{Low-pressure approximations}

If the pressure is not too high, the strangelet crystal consists of large Wigner-Seitz cells $\left(R_{\text {cell }} \gg R\right)$. In this regime one can obtain approximate analytic expressions for the equation of state of the crystal by assuming that the electrons have a roughly constant density outside the strangelet. We give these expressions below, and in later sections we use them to calculate mass radius relations for large strangelet dwarf stars. However, we expect these approximations break down at ultra-low pressures, when the cell size becomes so large that screening cannot be ignored, and the electrons are clumped around the strangelets, forming atoms, rather then being roughly uniformly distributed between the strangelets. This will happen when $R_{\text {cell }}$ approaches the Bohr radius $a_{0}=1 /\left(\alpha m_{e}\right)$, i.e. when $p_{\text {cell }} \lesssim \alpha^{5} Z^{5 / 3} m_{e}^{4} \approx$ $\left(10^{-12} \mathrm{MeV}^{4}\right) Z^{5 / 3}$. At these ultra-low pressures one should use an atomic matter equation of state: we do not do this, since we expect it will only affect a very small surface layer of the star, without any appreciable effect on the mass-radius relationship.

The equation of state $\varepsilon\left(p_{\text {cell }}\right)$ is found by writing the energy density $\varepsilon$ of the cell and its pressure as a function of the size of the cell. For now we will treat the size $R$ and charge $Z$ of the central strangelet as unknowns; later we will estimate their values.

Since the pressure inside a large cell is very low the energy density of the quark matter is approximately $n \mu_{\text {crit }}$, so

$$
\varepsilon \approx n \mu_{\text {crit }} \frac{R^{3}}{R_{\text {cell }}^{3}}
$$

To obtain the pressure at the edge of the cell we need to estimate the density distribution of the electrons outside the strangelet.

\section{Constant potential approximation}

The simplest approximation is to ignore screening, taking the electron Fermi momentum $k_{F e}$ to be independent of $r$ outside the strangelet (Sec. I of Ref. 33). Imposing neutrality of the cell fixes the Fermi momentum of the electrons,

$$
k_{F e}^{3}=\frac{9 \pi Z}{4 R_{\text {cell }}^{3}} .
$$

Using (17), we obtain the equation of state $\varepsilon\left(p_{\text {cell }}\right)$ of the strangelet crystal

$$
\varepsilon \approx \mu_{\mathrm{crit}} n \frac{4\left(k_{F e} R\right)^{3}}{9 \pi Z}
$$

where we use (7) to relate the electron Fermi momentum to $p_{\text {cell }}$.

Because the constant potential approximation gives a fairly simple expression we can use it to understand how the strangelet crystal EoS depends on the parameters of the quark matter EoS, and hence how the $M(R)$ curve for strangelet dwarf stars depends on those parameters. Note that in 19 the dependence of the energy density on the pressure is via a universal and monotonically increasing function $k_{F e}(p)$; dependence on the quark matter parameters enters via the factor that multiplies this function. To make the dependence on quark matter parameters explicit we use results for $R$ and $Z$ from Sec. III C 3 below, and rewrite 19 for the EoS of the strangelet crystal as

$$
\begin{aligned}
\varepsilon\left(p_{\text {cell }}\right) & \sim S\left(k_{F e}\left(p_{\text {cell }}\right)\right)^{3} \\
S & =\frac{\mu_{\text {crit }} n}{3 \pi^{2} n_{Q} \xi\left(x_{0}(\bar{\sigma})\right)}
\end{aligned}
$$

where all dependence on the quark matter parameters comes through the prefactor $S$, which has units of energy. $S$ can be explicitly obtained using (25), (27), and (28) for the $\xi$ function. One could informally think of $S$ as a "softness" parameter of the strangelet crystal EoS: as $S$ increases, the pressure becomes a more slowly-rising function of energy density. We expect that softer equations of state will yield smaller stars with lower maximum masses. In Table [1] we give the value of $S$ for a range of values of the parameters of the underlying quark matter EoS.

At low enough pressures, the electrons become nonrelativistic. Then $p_{\text {cell }} \approx k_{F e}^{5} /\left(15 \pi^{2} m_{e}\right)$, and 19 simplifies to an analytic expression for the equation of state,

$$
\varepsilon_{N R} \approx \frac{4 R^{3}}{3 Z}\left(\frac{125 \pi}{9} m_{e}^{3}\right)^{1 / 5} n \mu_{\mathrm{crit}} p_{\mathrm{cell}}^{3 / 5}
$$

This is a reasonable approximation when $k_{F e} \lesssim m_{e}$, i.e. when $p_{\text {cell }} \lesssim m_{e}^{4} /\left(24 \pi^{2}\right) \approx 0.0003 \mathrm{MeV}^{4}$. However, as we will see below, at the very lowest pressures the constant potential approximation becomes inaccurate. 


\section{Coulomb potential approximation}

We can improve on the constant potential approximation by including the Coulomb energy of the electrons in the calculation of the pressure. The equation of state is still given by (19), but now the relationship between $p_{\text {cell }}$ and $k_{F e}$ is modified by the addition of a Coulomb energy term (Ref. [33], (5)), yielding

$$
p_{\text {cell }}=p_{e^{-}}-\frac{\alpha}{5}\left(\frac{Z^{2}}{18 \pi^{7}}\right)^{1 / 3} k_{F e}^{4} .
$$

Unlike the constant potential approximation, the Coulomb potential approximation gives an energy density that goes to a non-zero value at zero pressure,

$$
\varepsilon_{\text {Coul }}(0)=\mu_{\text {crit }} n \frac{2 Z\left(\alpha m_{e} R\right)^{3}}{3 \pi^{2}} .
$$

Comparing with (17) we see that this corresponds to the energy of cells with size of order $1 /\left(\alpha m_{e}\right) \sim 10^{-10} \mathrm{~m}$. This is the energy density of a lattice of zero-pressure atomic matter with strangelets in place of nuclei, which is a reasonable guess for the low-pressure configuration of strangelets. We will therefore use the Coulomb approximation as the low-pressure extension of our equation of state. As we will see, this leads to a "planet" branch in the mass-radius relation for configurations of strange matter.

\section{Radius and charge of strangelet at low pressure}

The low-pressure approximation expressions given above depend on the size $R$ and charge $Z$ of the strangelet at the center of a large cell. This is approximately an isolated strangelet, whose radius can be calculated by minimizing the isolated strangelet free energy given in eqn (25) of Ref. [13,

$$
\overline{\Delta g}(x)=-\frac{3}{2} \frac{x-\tanh x}{x^{3}}+\frac{3 \bar{\sigma}}{x},
$$

where $x$ is the radius of the strangelet in units of $\lambda_{D}$, and

$$
\bar{\sigma}=\frac{\sigma}{4 \pi \alpha n_{Q}^{2} \lambda_{D}^{3}} .
$$

So the strangelet radius $R$ as a function of the parameters of the quark matter equation of state is

$$
R=x_{0} \lambda_{D}, \quad \text { where } \quad \frac{d \overline{\Delta g}}{d x}\left(x_{0}\right)=0 .
$$

We are interested in values of $\bar{\sigma}$ up to 0.13 , since for higher surface tension the strangelet crystal is no longer stable [13. An approximate expression for the solution to 26 , accurate to about $0.2 \%$ for $\bar{\sigma} \lesssim 0.13$, is

$$
x_{0}^{\text {approx }}=\left(\frac{15 \bar{\sigma}}{2}\right)^{1 / 3}+\frac{2.174 \bar{\sigma}}{1-3.982 \bar{\sigma}},
$$

where the first term is the leading-order analytic expression for $x_{0}$ in the limit of small $\bar{\sigma}$.

The charge $Z$ of the central strangelet is given by eqn. (17) of Ref. [13, which can be written

$$
\begin{aligned}
Z & \approx \frac{4}{3} \pi R^{3} n_{Q} \xi\left(R / \lambda_{D}\right), \\
\xi(x) & \equiv \frac{3}{x^{3}}(x-\tanh x),
\end{aligned}
$$

where $\xi$ is a correction for the effects of screening inside the quark matter; it is an even function with $\xi(0)=1$.

\section{NUMERICAL RESULTS}

\section{A. Range of parameters studied}

Our assumption that the strange matter hypothesis is valid requires that $\mu_{\text {crit }}$ must be less than the quark chemical potential of nuclear matter, about $310 \mathrm{MeV}$, so we fix $\mu_{\text {crit }}=300 \mathrm{MeV}$. The value of $\mu$ inside our strange matter lumps will always be within a few $\mathrm{MeV}$ of $\mu_{\text {crit }}$, because if the surface tension is small enough to favor the strangelet crystal it will not cause significant compression.

We will perform calculations for $\lambda_{D}=4.82 \mathrm{fm}$ and $\lambda_{D}=6.82 \mathrm{fm}$, corresponding to $\chi_{Q} \approx 0.2 \mu_{\text {crit }}^{2}$ (appropriate for unpaired quark matter $(6)$ ) and $\chi_{Q} \approx 0.1 \mu_{\text {crit }}^{2}$ (appropriate for 2SC quark matter [13]).

Typical values of $n_{Q}$ will be around $0.05 \mu_{\text {crit }} m_{s}^{2}$ (6), and a reasonable range would correspond to varying $m_{s}$ over its physically plausible range, from about 100 to 300 $\mathrm{MeV}$. (To have strange matter in the star, $m_{s}$ must be less than $\mu_{\text {crit }}$.) In this paper we use $n_{Q}=0.0445,0.0791$, and $0.124 \mathrm{fm}^{-3}$, which would correspond to $m_{s}=150$, 200 , and $250 \mathrm{MeV}$ in (6).

There is another widely-discussed phase of quark matter, the color-flavor locked (CFL) phase, but it is a degenerate case where $n_{Q}=\chi_{Q}=0$. CFL strangelets have a surface charge, but it does not arise from the mechanism studied here, Debye screening, and has a different dependence on the size of the strangelet [26]. We hope to study CFL strangelet matter in a separate work.

\section{B. Testing approximations to the equation of state}

In Fig. 11 we show the equation of state for the strangelet crystal, for critical quark chemical potential $\mu_{\text {crit }}=300 \mathrm{MeV}$, quark matter screening distance $\lambda_{D}=6.82 \mathrm{fm}$, quark charge density parameter $n_{Q}=$ $0.0791 \mathrm{fm}^{-3}$, and quark matter surface tension $\sigma=$ $1.0 \mathrm{MeVfm}^{-2}$. The dots were obtained numerically following the procedure of Sec. IIIB. The solid line is the Coulomb-potential approximation (Sec. III C 2). On this plot the constant potential approximation (Sec. III C 1 ) 


\begin{tabular}{ccccccc}
$\lambda_{D}$ & $n_{Q}$ & $\sigma_{\text {crit }}$ & \multicolumn{4}{c}{ Softness prefactor $S(\mathrm{MeV})$ at } \\
$(\mathrm{fm})$ & $\left(\mathrm{fm}^{-3}\right)$ & $\left(\mathrm{MeV} \mathrm{fm}^{-2}\right)$ & $\sigma=0.3$ & $\sigma=1.0$ & $\sigma=3.0$ & $\sigma=10.0$ \\
\hline 4.82 & 0.0445 & 0.533 & 345 & - & - & - \\
4.82 & 0.0791 & 1.69 & 158 & 202 & - & - \\
4.82 & 0.124 & 4.12 & 94 & 104 & 140 & - \\
6.82 & 0.0445 & 1.51 & 280 & 367 & - & - \\
6.82 & 0.0791 & 4.8 & 146 & 161 & 206 & - \\
6.82 & 0.124 & 11.6 & 90 & 95 & 105 & 155 \\
\hline
\end{tabular}

TABLE I: Softness prefactor 20 of the strangelet crystal for various quark matter equation of state. The first two columns, $\lambda_{D}$ and $n_{Q}$, specify the quark matter equation of state 3 (via (5)). The third column gives the maximum surface tension for which a strangelet crystal will occur (4). The last four columns give the softness prefactor $S$ for different values of the surface tension $\sigma$ (given in $\mathrm{MeV} \mathrm{fm}^{-2}$ ) of the interface between quark matter and vacuum.

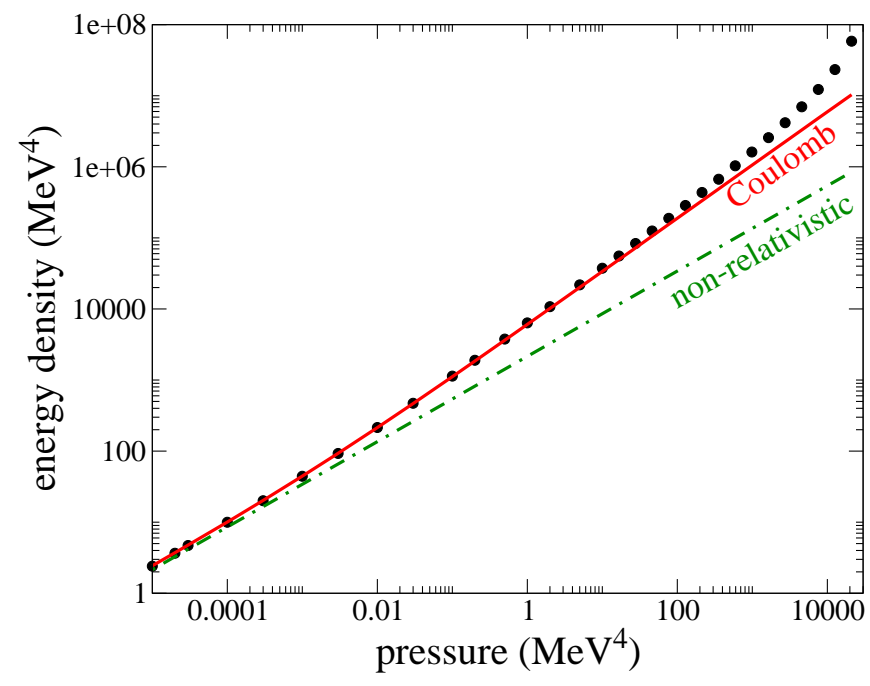

FIG. 1: Equation of state of the mixed phase (strangelet crystal) for strange matter with $\mu_{\text {crit }}=300 \mathrm{MeV}, \lambda_{D}=6.82 \mathrm{fm}$, $n_{Q}=0.0791 \mathrm{fm}^{-3}, \sigma=1.0 \mathrm{MeVfm}^{-2}$. The dots were obtained numerically following the procedure of Sec. IIIB The solid line is the Coulomb-potential approximation (Sec. III C 2. The dashed line is the non-relativistic electron (ultra-low pressure) limit 21. Above $p \approx 20000 \mathrm{MeV}^{4}$, uniform quark matter becomes favored over the mixed phase.

line would be indistinguishable from the Coulombpotential line, so we do not show it. The dot-dashed line is the non-relativistic electron (ultra-low pressure) limit 21] of the constant potential approximation. Above $p \approx 20000 \mathrm{MeV}^{4}$, uniform quark matter becomes favored over the mixed phase. On this very expanded logarithmic scale, the Coulomb approximation appears reasonably accurate up to pressures of order $1 \mathrm{MeV}$.

To achieve more discrimination between the different approximations, we show in Fig. 2 a magnified version of the low-pressure end of the plot in Fig. 1, where we have divided out the non-relativistic scaling of the energy density, $\varepsilon \sim p^{3 / 5}$. We can see that, down to the lowest pressures for which we can perform the numerical Wigner-Seitz calculation of the equation of state, the

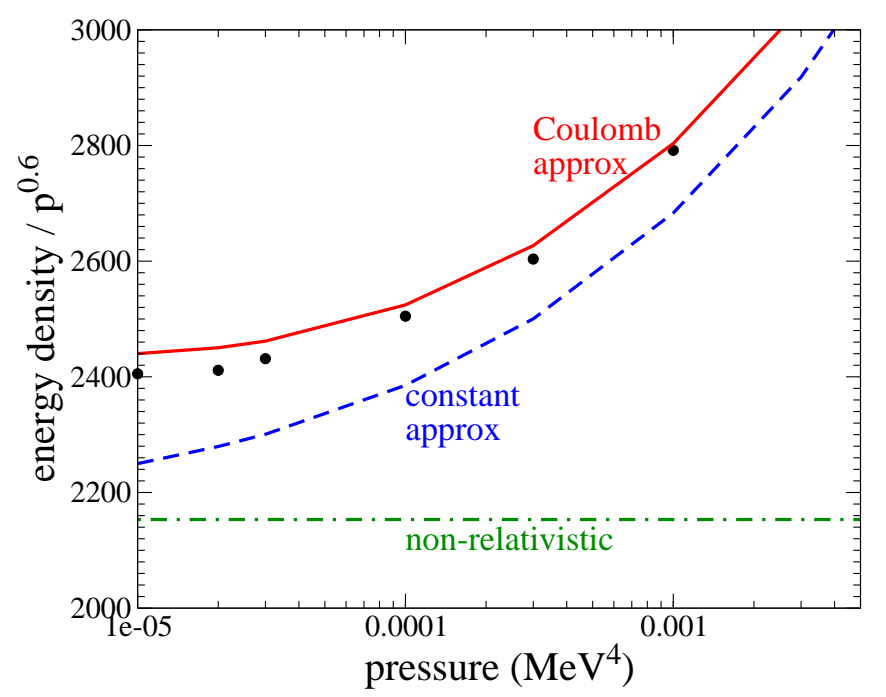

FIG. 2: Equation of state of the mixed phase for the same parameters as in Fig. 11 zoomed in on the low pressure region, and with the energy density divided by $p^{0.6}$. The dots were obtained numerically following the procedure of Sec. III B The Coulombpotential approximation (Sec. III C 2 is the most accurate, followed by the constant-potential approximation (Sec. III C 1), and then the non-relativistic electron approximation 21).

Coulomb approximation gives the most accurate semianalytic approximation, although the constant potential approximation is accurate to within about $10 \%$.

We then have to decide which approximation to use for lower pressures, where numerical calculations are not available. In the low-pressure limit, the Coulomb approximation to $\varepsilon(p)$ tends to a fixed value, while the constant and nonrelativistic approximations to $\varepsilon(p)$ tend to zero as $p^{3 / 5}$. So in Fig. 2 the Coulomb approximation will diverge at $p \ll 10^{-5} \mathrm{MeV}^{4}$, while the constant and nonrelativistic approximations will tend to the same constant value. As discussed in Sec. III C2, it seems reasonable to expect that at the lowest pressures there will be a crystal of "strange atoms", each consisting of electrons bound to a strangelet, and the Coulomb approximation gives a reasonable estimate of the energy density of such matter, so 


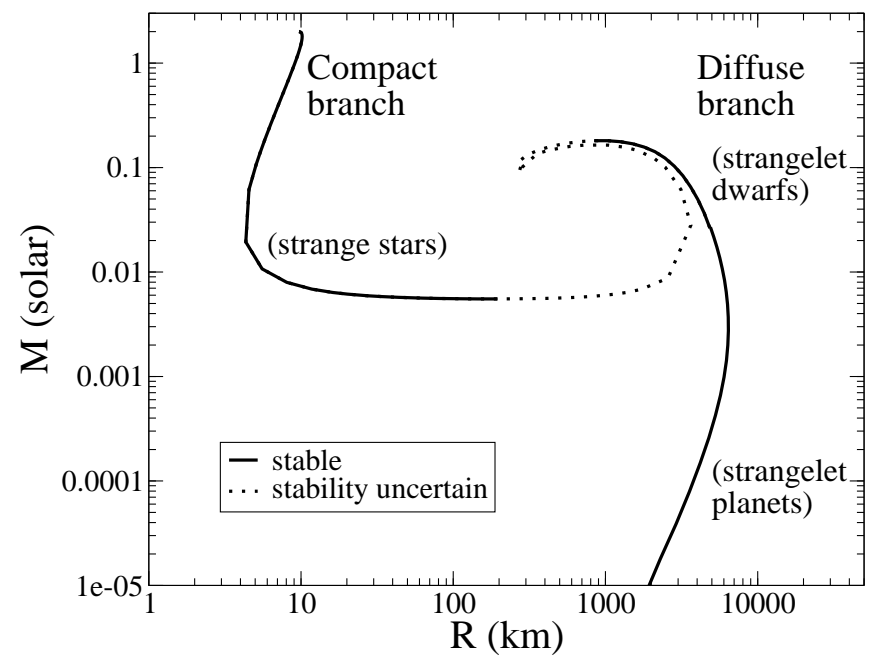

FIG. 3: The full mass-radius curve for stars made of quark matter with the equation of state plotted in Fig. 1. using the Coulomb approximation 22 to extrapolate to lower pressures. The compact branch contains strange stars with a strangelet crystal crust. The diffuse branch contains stars consisting entirely of strangelet crystal matter. Solid lines represent configurations that are stable; stability of the other branches is discussed in the text.

at low pressure we will use the Coulomb approximation.

\section{Mass-radius relation of strange stars}

In Fig. 3 we show the full mass-radius curve for stars made of quark matter with the equation of state plotted in Fig. 1. The compact branch contains strange stars with a strangelet crystal crust. The diffuse branch contains stars consisting entirely of strangelet crystal matter. It includes two segments: the lighter one is planets of dilute strange matter whose the mass increases with radius. This joins to the strangelet dwarf branch where the mass decreases with radius as the strangelet crystal is compressed by the pressure due to gravity. We use the numerically calculated equation of state (Sec. III) except that at very low pressure (the planetary branch) the Wigner-Seitz cells become so large that our numerical methods break down, so as discussed in Sec. IIIC2 we use the Coulomb approximation 22 to extrapolate down to zero pressure.

Fig. 3 shows the whole $M(R)$ curve, not all of which corresponds to stable configurations. The usual stability criterion for stars 34 is that one radial mode becomes either stable or unstable at each extremum in the $M(R)$ function. A stable mode becomes unstable at each extremum where the curve bends counterclockwise as the central density increases; a stable mode becomes unstable at each extremum where the curve bends clockwise as the central density increases. However, Glendenning et.al. 35 report that at some extrema there is no change in stability: the squared frequency of one of

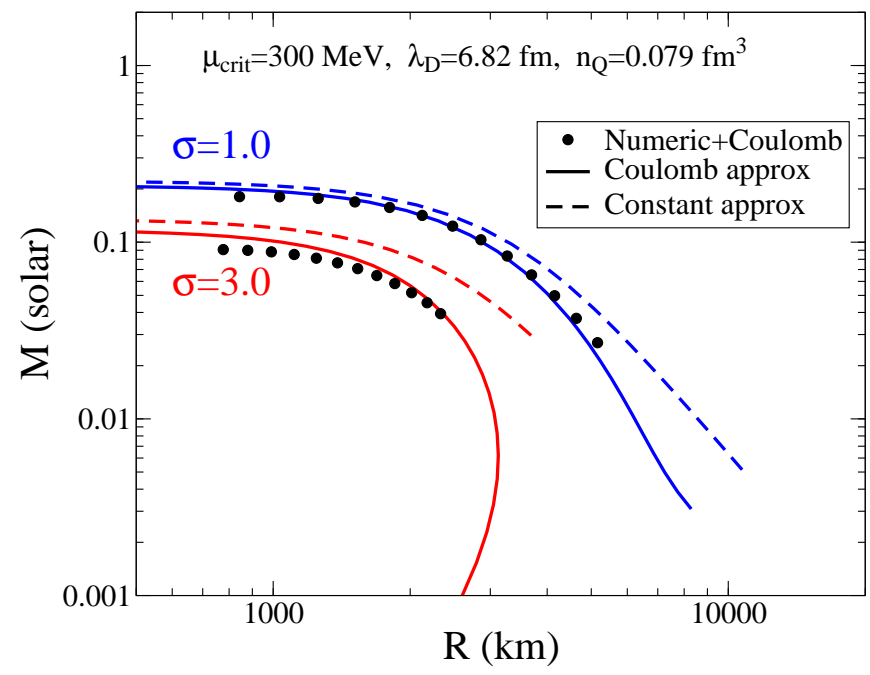

FIG. 4: Mass-radius relation for strangelet dwarfs made of strangelet crystal matter, comparing different approximations to the equation of state. Upper (blue) curves are for the same parameters as in Figs. 1 and 2 Lower (red) curves are for a larger surface tension, $\sigma=3 \mathrm{MeVfm}^{-2}$. The dots were obtained using the full numerical equation of state (Sec. III B). The solid lines use the Coulomb-potential approximation (Sec. III C 2), and the dashed lines use the constantpotential approximation 19 .

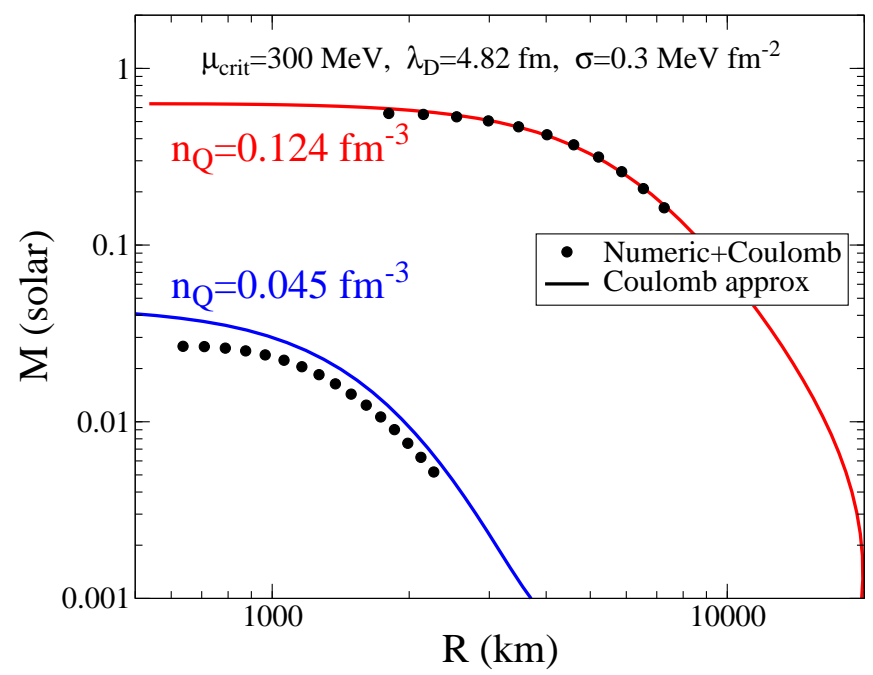

FIG. 5: Mass-radius relation for strangelet dwarfs made of strangelet crystal matter, comparing different approximations to the equation of state.

the fundamental radial modes may touch zero, but not change sign. We defer a detailed study of the stability of radial modes of strange stars to future work, and in Fig. 3 we show as "stable" (solid curves) the parts of the $M(R)$ curve that both Ref. [34] and Ref. [35] agree are stable. We note that Ref. [35] is a study of stars that have a core of uniform strange matter surrounded by a crust of nuclear matter: these are similar to the configurations along the dashed part of the mass-radius curve in Fig. 3, where 
we have a core of uniform strange matter surrounded by a crust of strangelets, with a density discontinuity at the boundary. If Ref. [35]'s stability argument is correct and applicable to our stars, then some of these configurations may also be stable. In the remainder of this paper we will focus on the strangelet dwarf branch, which consists of a simple crystal of stranglets with no uniform core, so there is no controversy about the appropriate stability criterion.

\section{Mass-radius relation of strangelet dwarfs}

To investigate the sensitivity of the masses and radii of strangelet dwarfs to the parameters of the quark matter equation of state, we show in Fig. 4 and 5 the strangelet dwarf part of the mass-radius curve, excluding the compact and planetary branches, for various values of the quark matter parameters.

In Fig. 4 we explore the effects of varying the surface tension, and we compare the different approximations to the equation of state. The upper curves are for the same equation of state as was shown in Fig. 1 and 2 the lower curves use a larger surface tension, $\sigma=3 \mathrm{MeVfm}^{-2}$. In both cases the solid curves are obtained from the Coulomb-potential approximation to the equation of state, and the dashed lines are obtained from the constant-potential approximation. The dots use the equation of state that is obtained numerically following the procedure of Sec. IIIB, except that at very low pressures, where the numerical calculation becomes too difficult, the Coulomb approximation is used.

We see that, as one might have expected from Fig. 1. using the Coulomb approximation over the entire pressure range of the mixed phase yields reasonably accurate results. However, as noted in Sec. III C 1, the constant potential approximation is still useful for gaining an understanding of how the $M(R)$ curve for strangelet dwarfs depends on the parameters of the EoS, because in the range of pressures that is important for strangelet dwarfs it gives a good indication of the $M(R)$ curve. (At ultralow pressures, relevant for the strange planet branch, this is no longer the case: one has to use the Coulomb approximation instead.) As discussed in Sec. IIIC1, the constant potential approximation to the EoS can be written in terms of a "softness prefactor" $S(20)$. To understand how the $M(R)$ curve in Fig. 4 changes with $\sigma$, note that $x_{0}(\bar{\sigma})$ is a monotonically increasing function and $\xi\left(x_{0}\right)$ is a monotonically decreasing function, so as the surface tension $\sigma$ increases at fixed values of the other parameters, the softness prefactor $S$ of the strangelet crystal EoS increases (one can see this in Table I). Since the EoS is becoming softer, the $M(R)$ curve moves down and to the left, giving smaller stars with a lower maximum mass.

In Fig. 5 we explore the effects of varying the charge density parameter $n_{Q}$ in (3) while keeping the other parameters constant. As in Fig. 4, solid lines are for the Coulomb approximation to the equation of state, dots are for the numerically calculated equation of state using the Coulomb approximation to extrapolate to the lowest pressures. We see that increasing $n_{Q}$ yields heavier, larger strangelet dwarf stars. Again, this can be understood in terms of the constant potential approximation and its softness prefactor $S$ 20). As $n_{Q}$ increases, it causes $S$ to decrease through two effects. Firstly via the explicit factor of $n_{Q}$ in the denominator of $20 p$, and secondly via the relationship (25) between $\sigma$ and $\bar{\sigma}$. The sensitivity of $S$ to changes in $n_{Q}$ can be seen in Table I for the two values of $n_{Q}$ used in Fig. 5 the values of $S$ are near the extremes of its range in the parameter set we studied: $S \approx 345$ and $S \approx 94$ for $n_{Q}=0.0445$ and $n_{Q}=0.124$ respectively. Consequently, the $M(R)$ curve for $n_{Q}=0.0445$ is characteristic of a soft equation of state, with low radius at a given mass and a low maximum mass, whereas the $M(R)$ curve for $n_{Q}=0.124$ is characteristic of a hard equation of state, with large radius at a given mass and a high maximum mass.

\section{DISCUSSION}

We have shown that, if the strange matter hypothesis is correct and the surface tension of the interface between strange matter and the vacuum is less than a critical value (4), there is at least one additional stable branch in the mass-radius relation for strange stars, corresponding to large diffuse objects that we call "strangelet dwarfs", consisting of a crystal of strangelets in a sea of electrons. This is easily understood, since if $\sigma<\sigma_{\text {crit }}$ then uniform strange matter is unstable at zero pressure, and undergoes charge separation to a crystal of positively-charged strangelets surrounded by electrons, just as normal matter at zero pressure is a mixed phase consisting of droplets of nuclear matter surrounded by electrons. Strangelet dwarfs are then the strange matter equivalent of white dwarfs.

We emphasize that in this low-surface-tension scenario, strange matter is not self bound. Like nuclear matter, it is only bound by gravitational forces. Every strange star will have a strangelet crystal crust, and strangelet dwarfs are those strange stars that are "all crust".

The natural production mechanism by which strangelet dwarfs might be produced is a collision between a strange star and another compact object. In such collisions, up to $0.03 M_{\odot}$ may be ejected [36], which is in the mass range we are predicting for strangelet dwarfs. There are two ways a collision could produce strangelet dwarfs. Firstly, part of the crust of the strange star might be ejected to become a isolated object, which would be a strangelet dwarf. Secondly, if a sufficiently light piece of the uniform quark matter core were ejected in the collision, it would be unable to exist on the compact branch, and would evaporate into a configuration on the diffuse branch. For example, for the equation of state studied in Fig. 3, the lightest compact configuration of strange matter is $0.0055 M_{\odot}$. 
A lighter piece of strange matter could only exist on the diffuse branch, and would spontaneously evaporate to become a strangelet dwarf. Strangelet dwarfs produced by these mechanisms could then bind gravitationally, to form heavier strangelet dwarfs.

It should be noted that our proposed mechanism for the production of strangelet dwarfs is also a mechanism for creating a diffuse cosmic flux of strangelets ("strangelet pollution"), which might be expected to convert all neutron stars to strange stars 37. Although observations of glitches and magnetar oscillations 38] seem consistent with some compact stars having nuclear matter crusts, there remains some uncertainty. Crystalline phases of quark matter could allow strange stars to glitch [39, and in our low-surface-tension scenario strange stars have crusts that could be hundreds of meters thick [14. A cosmic flux of strangelets may seem unlikely but until it is ruled out experimentally (as may happen soon from the AMS experiment [40]) it remains useful to analyze the full observational consequences of the strange matter hypothesis.

Our analysis assumes that at any given pressure the strangelet crystal consists of the most energetically favorable strangelet configuration (in terms of strangelet size and charge and cell size). However, other configurations will in general be metastable with long lifetimes. If one compresses a piece of strangelet crystal then the charge of the strangelets can readily change via absorption or emission of electrons, but it is very difficult for the quark matter to rearrange itself in to strangelets of the now-energetically-favored size: it is more likely that the strangelets will stay the same size and the radial density profile of the electrons will change. The sizes of the strangelets will be determined more by the history of the object than by the pressure. Taking this point further, it is quite possible to have a crystal consisting of a mixture of strangelets and ordinary nuclei, held apart by their electrostatic repulsion but also bound together in to a crystal by the degenerate electron gas that neutralizes them, forming a hybrid strangelet/white dwarf star.

Detection of strangelet dwarfs requires an observation method that can find non-luminous objects with typical masses of $10^{-5}$ to $10^{-1} M_{\odot}$ and radii in the range 500 to $5000 \mathrm{~km}$. An example is gravitational microlensing surveys, such as those conducted by the Microlensing Observations in Astrophysics (MOA) and the Optical Gravitational Lensing Experiments (OGLE) groups, which look for lensing events in the galactic bulge, and are capable of detecting Jupiter-mass objects. It is intriguing that such surveys now report the existence of an abundant population of unbound distant planetary masses, suggesting that such objects may be twice as common as main sequence stars [8]. Although models of planet formation indicate that mechanisms exist for unbinding planets through disk instabilities and planet interactions [41], we suggest that a possible alternative is formation of strange dwarfs from matter ejected in strange star mergers. One would expect that sometimes a strangelet dwarf produced in a merger might be unable to escape the gravitational field of the remaining compact object, and this would explain the presence of dense planet-mass objects in the vicinity of compact stars. An example is the millisecond pulsar PSR J1719-1438, which has a Jupiter-mass companion whose inferred central density $\left(\rho>23 \mathrm{~g} \mathrm{~cm}^{-3}\right)$ is far in excess of what is expected in a planet [42. We expect that in the near future further light will be cast on this question, as microlensing surveys help us better understand the distribution of planetary mass compact objects and as strategies are devised to provide information about both mass and radius.

\section{Acknowledgments}

We thank E. Agol and F. Weber for useful discussions. This research was supported in part by the Offices of Nuclear Physics and High Energy Physics of the Office of Science of the U.S. Department of Energy under contracts \#DE-FG02-91ER40628, \#DE-FG02-05ER41375, and by the DoE Topical Collaboration "Neutrinos and Nucleosynthesis in Hot and Dense Matter", contract \#DE-SC0004955.
[1] A. R. Bodmer, Collapsed nuclei, Phys. Rev. D4 (1971) 1601-1606.

[2] E. Witten, Cosmic Separation of Phases, Phys. Rev. D30 (1984) 272-285.

[3] E. Farhi and R. L. Jaffe, Strange Matter, Phys. Rev. D30 (1984) 2379.

[4] F. Weber, Strange quark matter and compact stars, Prog. Part. Nucl. Phys. 54 (2005) 193-288, [astro-ph/0407155].

[5] A. R. Zhitnitsky, 'Nonbaryonic' dark matter as baryonic color superconductor, JCAP 0310 (2003) 010, [hep-ph/0202161].

[6] S. Banerjee et. al., Massive compact halo objects from the relics of the cosmic quark-hadron transition, Mon. Not.
Roy. Astron. Soc. 340 (2003) 284, [astro-ph/0211560].

[7] M. M. Forbes, K. Lawson, and A. R. Zhitnitsky, The electrosphere of macroscopic 'quark nuclei': A source for diffuse $\mathrm{MeV}$ emissions from dark matter, arXiv: 0910.4541.

[8] T. Sumi, K. Kamiya, D. P. Bennett, I. A. Bond, F. Abe, C. S. Botzler, A. Fukui, K. Furusawa, J. B. Hearnshaw, Y. Itow, P. M. Kilmartin, A. Korpela, W. Lin, C. H. Ling, K. Masuda, Y. Matsubara, N. Miyake, M. Motomura, Y. Muraki, M. Nagaya, S. Nakamura, K. Ohnishi, T. Okumura, Y. C. Perrott, N. Rattenbury, T. Saito, T. Sako, D. J. Sullivan, W. L. Sweatman, P. J. Tristram, A. Udalski, M. K. Szymański, M. Kubiak, G. Pietrzyński, R. Poleski, I. Soszyński, Ł. Wyrzykowski, K. Ulaczyk, and 
Microlensing Observations in Astrophysics (MOA) Collaboration, Unbound or distant planetary mass population detected by gravitational microlensing, Nature (London) 473 (May, 2011) 349-352, [arXiv: 1105.3544].

[9] C. Alcock, E. Farhi, and A. Olinto, Strange stars, Astrophys. J. 310 (1986) 261-272.

[10] M. Stejner and J. Madsen, Gaps below strange star crusts, Phys. Rev. D72 (2005) 123005, [astro-ph/0512144].

[11] V. V. Usov, Low-mass normal-matter atmospheres of strange stars and their radiation, Astrophys. J. 481 (1997) L107-L110, [astro-ph/9703037].

[12] P. Jaikumar, S. Reddy, and A. W. Steiner, The strange star surface: A crust with nuggets, Phys. Rev. Lett. 96 (2006) 041101, [nucl-th/0507055].

[13] M. G. Alford, K. Rajagopal, S. Reddy, and A. W. Steiner, The stability of strange star crusts and strangelets, Phys. Rev. D73 (2006) 114016, [hep-ph/0604134].

[14] M. G. Alford and D. A. Eby, Thickness of the strangelet-crystal crust of a strange star, Phys. Rev. C78 (2008) 045802, [arXiv:0808.0671].

[15] A. Y. Potekhin and G. Chabrier, Equation of state of fully ionized electron-ion plasmas. II. extension to relativistic densities and to the solid phase, Phys. Rev. E 62 (Dec., 2000) 8554-8563, [astro-ph/0009261].

[16] D. G. Ravenhall, C. J. Pethick, and J. R. Wilson, Structure of matter below nuclear saturation density, Phys. Rev. Lett. 50 (1983) 2066-2069.

[17] N. K. Glendenning, First order phase transitions with more than one conserved charge: Consequences for neutron stars, Phys. Rev. D46 (1992) 1274-1287.

[18] M. Alford, C. Kouvaris, and K. Rajagopal, Evaluating the gapless color-flavor locked phase, Phys. Rev. D71 (2005) 054009, [hep-ph/0406137].

[19] M. G. Alford, K. Rajagopal, and F. Wilczek, Color-flavor locking and chiral symmetry breaking in high density QCD, Nucl. Phys. B537 (1999) 443-458, [hep-ph/9804403].

[20] R. C. Tolman, Static solutions of Einstein's field equations for spheres of fluid, Phys. Rev. 55 (1939) 364-373.

[21] J. R. Oppenheimer and G. M. Volkoff, On Massive neutron cores, Phys. Rev. 55 (1939) 374-381.

[22] T. Maruyama, S. Chiba, H.-J. Schulze, and T. Tatsumi, Hadron-quark mixed phase in hyperon stars, Phys. Rev. D76 (2007) 123015, [arXiv:0708.3277].

[23] J. Madsen, Shell model versus liquid drop model for strangelets, Phys. Rev. D50 (1994) 3328-3331, [hep-ph/9407314].

[24] P. Amore, M. C. Birse, J. A. McGovern, and N. R. Walet, Colour superconductivity in finite systems, Phys. Rev. D65 (2002) 074005, [hep-ph/0110267].

[25] J. Madsen, Intermediate mass strangelets are positively charged, Phys. Rev. Lett. 85 (2000) 4687-4690, [hep-ph/0008217].

[26] J. Madsen, Color-flavor locked strangelets, Phys. Rev. Lett. 87 (2001) 172003, [hep-ph/0108036].
[27] J. Madsen, Universal charge-radius relation for subatomic and astrophysical compact objects, Phys. Rev. Lett. 100 (2008) 151102, [arXiv:0804.2140].

[28] M. Oertel and M. Urban, Surface effects in color superconducting strangelets and strange stars, Phys. Rev. D77 (2008) 074015, [arXiv:0801.2313].

[29] M. B. Christiansen and N. K. Glendenning, Finite size effects and the mixed quark-hadron phase in neutron stars, Phys. Rev. C56 (1997) 2858-2864, [astro-ph/9706056].

[30] M. B. Christiansen and J. Madsen, Inhomogeneity scale from the cosmological quark-hadron transition, J. Phys. G23 (1997) 2039-2046.

[31] M. S. Berger and R. L. Jaffe, Radioactivity in strange quark matter, Phys. Rev. C35 (1987) 213-225.

[32] M. S. Berger and R. L. Jaffe, Erratum: Radioactivity in strange quark matter, Phys. Rev. C 44 (Jul, 1991) 566.

[33] E. E. Salpeter, Energy and Pressure of a Zero-Temperature Plasma, Astrophys. J. 134 (1961) 669-682.

[34] J. M. Bardeen, K. S. Thorne, and D. W. Meltzer, A catalogue of methods for studying the normal modes of radial pulsation of general-relativistic stellar models, Astrophys. J. 145 (Aug., 1966) 505-+.

[35] N. Glendenning, C. Kettner, and F. Weber, A Possible new class of dense white dwarfs, Phys.Rev.Lett. 74 (1995) 3519-3521.

[36] A. Bauswein, H.-T. Janka, R. Oechslin, G. Pagliara, I. Sagert, et. al., Mass Ejection by Strange Star Mergers and Observational Implications, Phys.Rev.Lett. 103 (2009) 011101, [arXiv:0812.4248].

[37] J. Friedman and R. Caldwell, Evidence against a strange ground state for baryons, Phys.Lett. B264 (1991) 143-148.

[38] A. L. Watts and S. Reddy, Magnetar oscillations pose challenges for strange stars, Mon. Not. Roy. Astron. Soc. 379 (2007) L63, [astro-ph/0609364].

[39] M. Mannarelli, K. Rajagopal, and R. Sharma, The Rigidity of crystalline color superconducting quark matter, Phys.Rev. D76 (2007) 074026, [hep-ph/0702021].

[40] J. Sandweiss, Overview of strangelet searches and Alpha Magnetic Spectrometer: When will we stop searching?, J.Phys.G G30 (2004) S51-S59.

[41] D. Veras, J. R. Crepp, and E. B. Ford, Formation, Survival, and Detectability of Planets Beyond $100 \mathrm{AU}$, Astrophys. J. 696 (May, 2009) 1600-1611, [arXiv:0902 .2779].

[42] M. Bailes, S. D. Bates, V. Bhalerao, N. D. R. Bhat, M. Burgay, S. Burke-Spolaor, N. DAmico, S. Johnston, M. J. Keith, M. Kramer, S. R. Kulkarni, L. Levin, A. G Lyne, S. Milia, A. Possenti, L. Spitler, B. Stappers, and W. van Straten, Transformation of a Star into a Planet in a Millisecond Pulsar Binary, Science 333 (Sept., 2011) 1717-, [arXiv:1108.5201]. 